\title{
Influence of Nasal Congestion on Respiratory Resistance Values
}

Awowale $A^{1}$, Johnson $A^{1 *}$ and Vossoughi $\mathbf{J}^{1,2}$

${ }^{1}$ Fischell Department of Bioengineering, University of Maryland, College Park, USA

${ }^{2}$ Engineering \& Scientific Research Associates, Olney, USA

\begin{abstract}
Nasal congestion is the fourth most common minor ailment presented in primary care and as such, a method to quantify the meaning of nasal congestion in order to enable evaluation of medicines catered to specific congestion levels can prove important. The Airflow Perturbation Device (APD) is a noninvasive respiratory diagnostic device that evaluates the respiratory resistance in humans. It measures the total respiratory resistance under normal breathing in less than one minute. This study involved using the APD to determine the influence of nasal congestion on respiratory resistance in a laboratory setting. A total of 25 volunteers volunteered for this study and it employed a standard subjective categorical scale for nasal congestion (i.e. No Congestion, Mild Congestion, Moderate Congestion, and Severe Congestion). The results show that resistance values increased with increased congestion levels. However, resistance values of the groups of volunteers for the various congestion categories overlapped, and there were no statistically significant values differentiating no congestion and mild congestion or moderate congestion and severe congestion.
\end{abstract}

Keywords: Airflow Perturbation Device (APD); Nasal congestion; Respiratory Resistance

\section{Introduction}

Nasal congestion is the fourth most common minor ailment presented in primary care and as such, a method to quantify its symptoms in order to enable evaluation of prescription medicines catered to specific congestion levels could prove important. The APD is a noninvasive respiratory diagnostic device that measures the total respiratory resistance under normal breathing in less than one minute. The APD works by periodically inserting an external resistance in the breathing flow pathway [1-4]. A rotating wheel in the flow path perturbs air flow and mouth pressure by a small amount (Figure 1). When this happens, the respiratory flow is reduced and the mouth pressure is increased. The APD senses these changes and is used to compute respiratory resistance.

Respiratory resistance is normally measured through the mouth, with a nose clip attached to the nose. An oronasal mask can be used to obtain combined mouth and nose resistance, or only nose resistance, if the mouth is closed. Data collected by the APD are analyzed in real time and displayed as averages of the last few perturbations. The APD separates the resistances during inhalation from those during

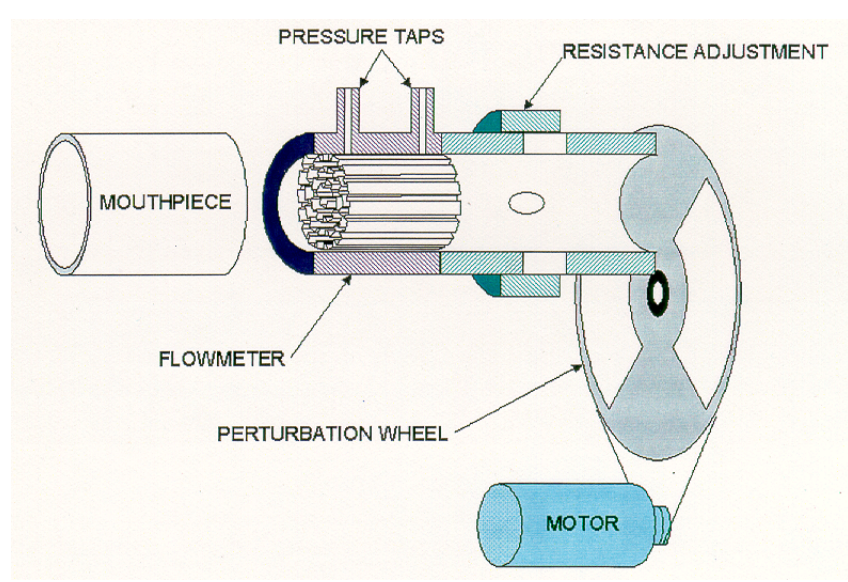

Figure 1: Schematic diagram of the APD. exhalation, which can be used as diagnostic information related to specific respiratory diseases.

\section{Methods and Materials}

\section{Subject recruitment}

A total of 25 subjects were tested in this study. Because human subjects were used in this study, approval came from the University of Maryland's Institutional Review Board (IRB). All subjects were required to sign informed consent documents before participating. A majority of the test subjects were undergraduate students known by the investigator and studying at the University of Maryland. Prior to the test, all subjects were given a brief introduction and a brief synopsis of the research project and testing procedure. In addition, each subject was asked brief questions regarding present and past health status. From this questionnaire, it was determined that six of the volunteers had a history of asthma and 13 had seasonal allergies (not necessarily active at the time of testing). There were no exclusion criteria because each subject was to rate nasal congestion according to subjective feeling. Likewise, it was not determined if their nasal passages were clear before testing, because, if they came to the lab with some preexisting nasal congestion, they could begin the study without starting at the No Congestion level. Each subject took about 45 minutes to an hour to complete the test.

\section{Testing}

The testing protocol consisted of four nasal congestion tests for each of the following: 1) no congestion, 2) mild congestion, 3) moderate congestion, and 4) severe congestion. Because the APD measures total respiratory resistance from airway openings to the chest wall surface, mouth only, nose only ( $\mathrm{N}$ condition), and nose and mouth

*Corresponding author: Johnson A, Professor Emeritus, University of Maryland Bioengineering 1429 ANSC, College Park, Maryland 20742, USA, Tel: 1-301-4051184; E-mail: artjohns@umd.edu

Received August 24, 2014; Accepted December 29, 2014; Published January 02,2015

Citation: Awowale A, Johnson A, Vossoughi J (2015) Influence of Nasal Congestion on Respiratory Resistance Values. J Pulm Respir Med 5: 228. doi:10.4172/2161. 105X.1000228

Copyright: ( 2015 Awowale A, et al. This is an open-access article distributed under the terms of the Creative Commons Attribution License, which permits unrestricted use, distribution, and reproduction in any medium, provided the original author and source are credited. 
Citation: Awowale A, Johnson A, Vossoughi J (2015) Influence of Nasal Congestion on Respiratory Resistance Values. J Pulm Respir Med 5: 228. doi:10.4172/2161-105X.1000228

Page 2 of 3

\begin{tabular}{|c|c|c|c|c|c|c|c|c|c|}
\hline & \multicolumn{3}{|c|}{ Mouth only with Nose Clip } & \multicolumn{3}{|c|}{ Nose only (N) } & \multicolumn{3}{|c|}{ Nose and Mouth (NM) } \\
\hline & Mean & Std Dev & Range & Mean & $\begin{array}{l}\text { Std Dev of all } \\
\text { measurements }\end{array}$ & Range & Mean & $\begin{array}{l}\text { Std Dev of all } \\
\text { measurements }\end{array}$ & Range \\
\hline No congestion & 4.21 & 1.15 & $1.61-6.52$ & 4.89 & 1.04 & $1.54-6.24$ & 4.63 & 0.95 & $1.78-6.62$ \\
\hline Mild congestion & & & & 5.54 & 1.31 & $1.46-7.82$ & 5.14 & 1.17 & $1.38-6.51$ \\
\hline Moderate congestion & & & & 6.00 & 1.38 & $1.77-8.53$ & 5.33 & 1.12 & $1.67-6.99$ \\
\hline Severe congestion & & & & 6.47 & 1.35 & $1.66-7.91$ & 5.58 & 1.11 & $1.49-6.83$ \\
\hline
\end{tabular}

Table 1: Average resistance values $\left(\mathrm{Cm} \mathrm{H}_{2} \mathrm{O} / \mathrm{Lps}\right)$ for different test conditions.

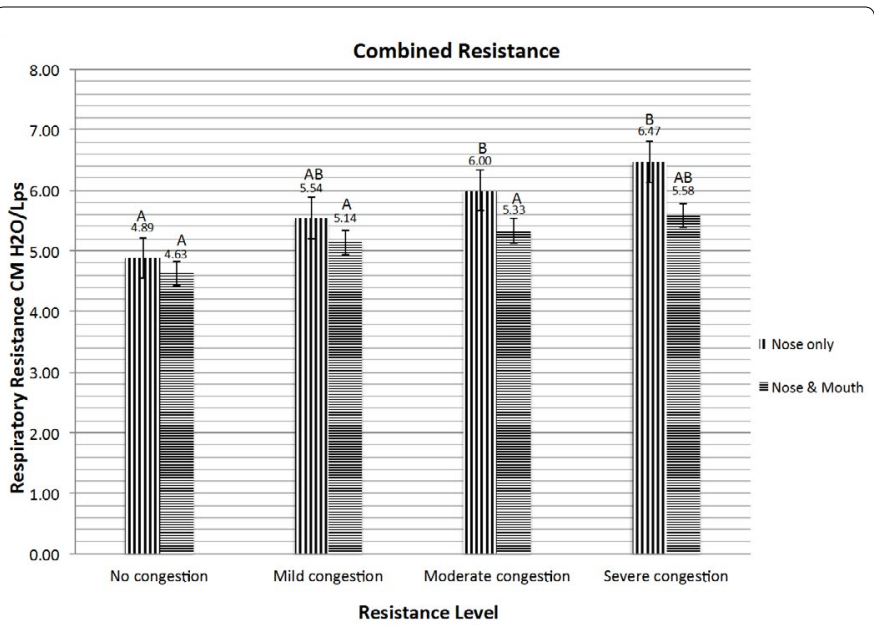

Figure 2: Average resistance values with different levels of congestion. Error bars represent standard error. Means with different letters are significantly different (Tukey's HSD, $\mathrm{P}<0.05$ ).

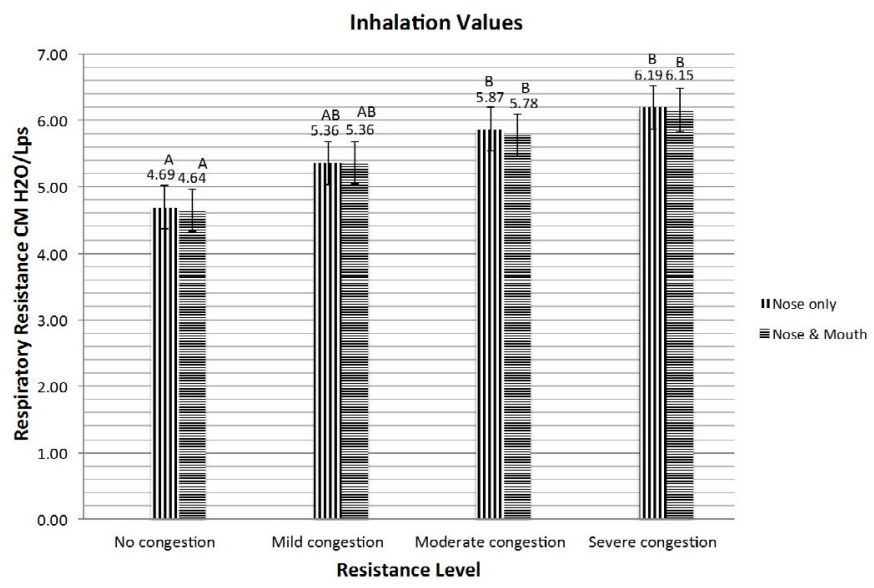

Figure 3: Inhalation resistance values with different levels of congestion. Error bars represent standard error. Means with different letters are significantly different (Tukey's HSD, $\mathrm{P}<0.05$ ).

(NM condition) together measurements were taken. Mouth only measurements were taken as a control base to compare with previous measurements, but did not add to nasal resistance evaluation. A nose clip was used to collect mouth only measurements and an oronasal mask was used to collect nose only and mouth and nose breathing measurements. To conduct this study in a laboratory setting, subjects were given commercially available nose cones to mimic congestion (Sinus Cones by Sanostec; Beverly Farms, MA). They were asked to insert cotton wads in the nose cones based on what they considered mild, moderate or severe congestion. The no congestion condition was measured with the nose cones inserted in the nostrils but without a cotton wad. Each subject made the judgment about congestion levels based on their own personal preferences. Five APD measurements for each congestion level were made. A total of 50 measurements per testing session were conducted.

\section{Statistical Analysis}

StatPlus:mac statistical analysis program was utilized to analyze the results. ANOVA was used to determine statistical significance in the group and Tukey's HSD test [5] at $\mathrm{p}<.05$ analysis was used to determine which groups were significantly different.

\section{Results}

It was observed from the data in Table 1 that that the average resistances generally increased with increased congestion levels but there are hardly any statistically significant differences between different congestion levels. $\mathrm{N}$ condition breathing for combined averages showed that no congestion was the only level that showed a statistically significant difference from other congestion levels (Table 1). For the NM condition, the only statistically significant different group was severe congestion (Figure 2). For NM condition inhalation values, no congestion showed a statistically significant difference from moderate congestion and severe congestion but mild congestion was not statistically significantly different from any of the other groups (Figure 3). For $\mathrm{N}$ condition exhalation values, no congestion was the only group that showed a statistically significant difference from the other groups (Figure 4). There existed no statistically significant difference between any of the NM condition congestion levels.

Of the subjects for this study, 14 were males and 11 were females. They ranged from 15 to 23 years old with a mean of 19.9 years. Statistical analysis (ANOVA) showed that there was no significant difference between resistances of males and females for the combined congestion as well as for inhalation and exhalation resistances measured separately (Supplementary Table of Appendix).

\section{Discussion}

Nasal congestion is the fourth most common minor ailment presenting in primary care, with 5.3 million consultations per year. As such, a method to quantify its symptoms in order to enable production of medicines catered to more specific congestion levels can prove important. In this study, we looked at the effects of varying levels of nasal congestion on respiratory resistance, and, with the APD, were able to observe a general trend between increased resistance values and increased levels of congestion. However, the fact that there were no clear (statistically significant) distinctions among congestion levels is not a good sign for development of a quantitative approach to nasal congestion drug evaluation. It is important to note that the subjects tested had a wide range of resistance without congestion. This can be due to the fact that nasal anatomy is characterized by a complex geometry and significant individual variation [6]. It had previously been reported that the least noticeable change in respiratory resistance is a proportion of the resistance present before the change [3,7], and 


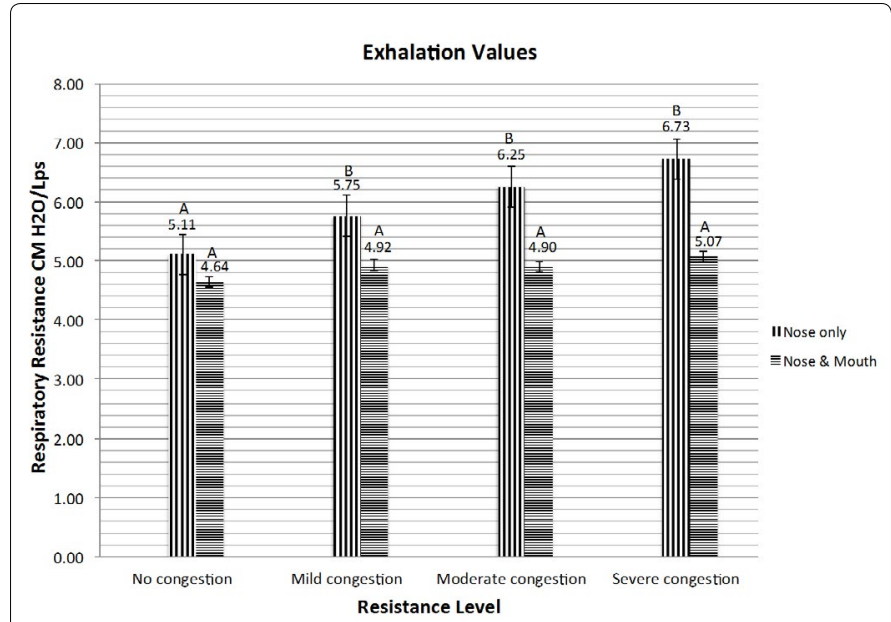

Figure 4: Exhalation resistance values with different levels of congestion. Error bars represent standard error. Means with different letters are significantly different (Tukey's HSD, $\mathrm{P}<0.05$ ).

generally conforms to Webers Law [8,9]. This would explain why some people notice any level of congestion more than others. Another difference may be due to the natural habits held by different individuals: some may just naturally breather harder than normal simply because they are thinking about their breathing. Other factors also include whether or not the subjects smoke, what medication they were on, allergies, if they had genetic respiratory illnesses, and even changes in posture. Therefore, subjective determination of congestion level probably reflects all of these individual differences.

The results of the experiment show that although there is an overall statistical significance in the data, statistical differences among groups vary. For example, for the average (inhalation and exhalation) nose only breathing, no congestion and moderate congestion when compared to each other, showed no statistically significant difference. However when compared to moderate or severe congestion, they did show statistical significance. This implies that pharmaceutical companies will have a hard time delineating between those congestion levels when creating quantitative evaluation of a congestion specific drug. Males and females had comparable average resistances. There were only three smokers tested in this study, and their resistance values were not significantly different from resistance values, averaged over all subjects.

As expected exhalation resistance values were greater than inhalation resistance values. This is because of the slightly narrowed respiratory airways in the lung as exhalation pressures external to the airways close them somewhat. Also as expected, resistances of mouth and nose taken together were less than resistances of nose only breathing because resistance of two parallel flow pathways is always less than that of a single pathway.

If this study had successfully shown that nasal congestion values could be quantified, then the results could have been of great interest to pharmaceutical companies developing congestion-relief medicines. Such results could also have been important for those prescribing medicines. In both of these cases, the treatment of congestion would have become much more precise. However, congestion levels are largely determined by subjective evaluation, meaning that they do not translate well from one individual to another. That is, then, the main conclusion of this study. Clinical treatment of congestion will continue as it is presently done.

\section{Conclusion}

The study was successful in demonstrating a trend between congestion and resistance: Higher congestion yielded higher resistance values, but subjective determinations of congestion levels did not separate neatly into levels of respiratory resistance.

\section{References}

1. Lausted G, Johnson A (1999) Respiratory resistance measured by an airflow perturbation device. Physiological Measurement 20: 21-35.

2. Silverman NK, Johnson AT (2005) Design for a Stand-Alone Airflow Perturbation Device. International Journal of Medical Implants and Devices 1: 139-148.

3. Johnson AT (2007) Biomechanics and Exercise Physiology: Quantitative Modelling. Taylor and Francis, Boca Raton., FL.

4. Haque T, Vossoughi J, Johnson AT, Bell-Farrell W, Fitzgerald T, et al. (2013) Resistance Measured by Airflow Perturbation Compared with Standard Pulmonary Function Measures. Open Journal of Respiratory Diseases 3: 6367.

5. Lowry, Richard (2000) One Way ANOVA - Independent Samples

6. Johnson AT, Jones SC, Pan JJ, Vossoughi J (2012) Variation of Respiratory Resistance Suggests Optimization of Airway Caliber. IEEE Transactions on Biomedical Engineering 59: 2355-2361.

7. Mahutte CK, Campbell EJM, Killian KJ (1983) Theory of Resistance Load Detection. Respiratory Physiology 55: 547-557.

8. Smith BD (1998) Psychology: Science and Understanding McGraw-Hill, New York.

9. Johnson AT (2011) Biology for Engineers. Taylor and Francis, Boca Raton. FL. 